\title{
Trastuzumab as a preoperative monotherapy does not inhibit HER2 downstream signaling in HER2-positive breast cancer
}

\author{
MAËVA LION ${ }^{1-3}$, ALEXANDRE HARLÉ $^{1-3}$, JULIA SALLERON $^{4}$, CAROLE RAMACCI $^{3}$, \\ MARIO CAMPONE ${ }^{5,6}$ and JEAN-LOUIS MERLIN ${ }^{1-3}$
}

\begin{abstract}
${ }^{1}$ Cellular Oncology Laboratory, Faculty of Pharmacy, Université de Lorraine, Nancy 54000;
${ }^{2}$ Centre National pour la Recherche Scientifique, Unité Mixte de Recherche 7039 CRAN, Université de Lorraine, Vandouvre-lès-Nancy 54500; ${ }^{3}$ Department of Biopathology; ${ }^{4}$ Biostatistics Unit, Institut de Cancérologie de Lorraine, Vandœuvre-lès-Nancy 54519; ${ }^{5}$ Institut National de la Santé et de la Recherche Médicale, Unité Mixte de Recherche 892, Nantes 44007; ${ }^{6}$ Department of Medical Oncology, Institut de Cancérologie de l'Ouest, St Herblain, Nantes 44800, France
\end{abstract}

Received September 4, 2015; Accepted May 23, 2016

DOI: $10.3892 /$ ol.2016.4891

\begin{abstract}
Human epidermal growth factor 2 (HER2) is overexpressed in $15-20 \%$ of breast carcinomas. The overexpression of HER 2 was previously associated with a poor prognosis until the development of the first anti-HER2 therapy, trastuzumab, which drastically improves the prognosis of HER2-overexpressing breast cancers. However, its mechanism of action remains not fully understood. Several studies have proposed that the behavior and mechanism of action of trastuzumab may be drastically altered in vitro and in vivo. The present study assesses the ability of trastuzumab to inhibit the phosphorylation of the key-proteins of phosphoinositide 3-kinase (PI3K)/protein kinase B (AKT)/mechanistic target of rapamycin and Ras/Raf/mitogen-activated protein kinase (MAPK) signaling pathways in vitro, in breast cancer cell lines and in tumor biopsies obtained from patients treated with trastuzumab preoperative monotherapy as part of the Unicancer GEP04 RADHER phase II clinical trial. HER2-positive SKBR3 and HER2-negative MCF-7 cell lines were exposed to trastuzumab for $72 \mathrm{~h}$. In total, 41 patients received trastuzumab alone for 6 weeks of preoperative treatment. Biopsies were collected at the baseline and at surgery. A total of 19 pairs of associated baseline and surgery tumor specimens were eligible for protein extraction and comparative phosphoprotein expression analysis, prior to and subsequent to treatment. The expression of phosphoproteins was quantitatively assessed using a multiplex immunoassay. In the SKBR3 cell line, a statistically significant decrease of the expression level of phosphorylated (p-)AKT, p-ribosomal protein S6
\end{abstract}

Correspondence to: Professor Jean-Louis Merlin, Department of Biopathology, Institut de Cancérologie de Lorraine, 6 Avenue de Bourgogne, CS 30519, Vandœuvre-lès-Nancy 54519, France E-mail: jl.merlin@nancy.unicancer.fr

Key words: breast cancer, trastuzumab, signaling pathways, phosphoproteins kinase B1, p-extracellular signal regulated kinase 1/2 and p-mitogen-activated protein kinase kinase 1 was observed after exposure to trastuzumab. In contrast, no statistically significant variations for levels expression of these phosphoproteins were observed in patients following treatment. The lack of downregulation of PI3K and MAPK pathways could probably be explained by the implementation of a predominant immunological mechanism of action for trastuzumab, a type of antibody-dependent cell-mediated toxicity, which has previously been reported in preoperative monotherapy settings. The present study confirms that trastuzumab involves various modes of action when assayed in vitro and used clinically.

\section{Introduction}

Breast cancer treatment has evolved in the last 20 years thanks to the better understanding of molecular mechanisms and the identification of numerous genomic alterations involved in the carcinogenesis process. This knowledge also allowed the identification of novel therapeutic targets and the development of numerous drugs specifically directed against these targets, called targeted therapies. The human epidermal growth factor 2 (HER2) oncoprotein is one of the first therapeutic targets identified for breast carcinoma treatment. This protein is a class 1 transmembrane receptor with tyrosine kinase activity and is involved in the oncogenic activation of numerous signaling pathways, including the mitogen-activated protein kinase (MAPK) and phosphoinositide 3-kinase (PI3K) pathways $(1,2)$. These pathways are important for cellular homeostasis and regulating the mechanisms of growth, differentiation, cell proliferation and apoptosis (3). The two pathways are frequently dysregulated in breast cancers due to mutations, deletions or rearrangements on the signaling proteins encoding genes (4).

The MAPK and PI3K pathways are kinase dependent. Activation of these kinases leads to the formation of their active phosphorylated states (5). The upregulation of signaling pathways and an increase in the phosphorylation of proteins that are involved in these pathways has been demonstrated in numerous cancers; this was particularly the case for phosphorylated-protein kinase B (p-AKT) in lung 
cancer, myeloid leukemia, prostate and breast cancers (6-8). Consequently, protein phosphorylation may be an indicator of signaling activity in tumors, and can be used to assess the activity of intracellular signaling pathways (9). Levels of phosphorylated forms of cells signaling proteins may be a prognostic biomarker and may predict the tumors sensitivity to treatment (10-12).

HER2 receptors are involved in tumor development and proliferation (13). Activating mutations on gene loci encoding the tyrosine kinase domain, or the overexpression of HER2 receptors, can lead to the upregulation or dysregulation of downstream signaling pathways (13). HER2 is overexpressed in $15-20 \%$ of breast carcinomas $(1,14)$.

The overexpression of HER2 was previously associated with a poor prognosis until the development of the first anti-HER2 therapy, trastuzumab, which drastically improves the progression-free and overall survival times of patients with HER2-overexpressing breast cancers (15).

Trastuzumab is an immunoglobulin G1 ( $\mathrm{IgG1}$ )-type humanized recombining monoclonal antibody $(\mathrm{mAb})$ directed against the extracellular domain of HER2 tyrosine kinase receptor $(16,17)$. This $m A b$ specifically binds the subdomain IV of HER2 extracellular domain, and through this molecular mechanism, trastuzumab activity is awaited by blocking HER2 downstream cell signaling (17-19). Due to the IgG1 backbone, trastuzumab also acts through antibody-dependent cell-mediated cytotoxicity (ADCC). The interaction of the Fc domain with Fc $\gamma$ receptor of immune cells, including natural killer cells and macrophages, leads to the activation of these cells and thus the lysis of cancer cells $(1,2,16,20-24)$.

Several studies have proposed that the behavior and mechanism of action of trastuzumab may be drastically altered in vitro and in vivo $(19,21)$, and that the relative involvement of signaling inhibition vs. ADCC could be an important determinant of the activity of trastuzumab (25).

The aim of the present study was to assess the ability of trastuzumab to inhibit the phosphorylation of the major proteins of the PI3K/AKT/mechanistic target of rapamycin (mTOR) and Ras/Raf/MAPK signaling pathways signaling pathways in vitro, in breast cancer cell lines and in tumor biopsies obtained from patients treated with preoperative trastuzumab monotherapy as part of the RADHER clinical trial.

\section{Materials and methods}

Cell lines. HER2-positive SKBR3 [American Type Culture Collection (ATCC) ${ }^{\circledR}$ HTB-30 ${ }^{\mathrm{TM}}$; ATCC, Manassas, VA, USA], and HER2 negative MCF-7 (ATCC ${ }^{\circledR} \mathrm{HTB}^{-22}{ }^{\mathrm{TM}}$; ATCC), cell lines were maintained in phenol red-free RPMI-1640 culture medium supplemented by $10 \%$ fetal calf serum (FCS; Dutscher, Brumath, France) in a $5 \% \mathrm{CO}_{2}$ atmosphere. The two cell lines were exposed to trastuzumab $\left(5.10^{-6} \mathrm{~mol} / \mathrm{l}\right)$ for $72 \mathrm{~h}$ in FCS-free medium.

Patients. All patients $(n=41)$ had non-metastatic early or locally-advanced HER2 breast carcinomas (T1-3, N0-2, M0) according to the TNM staging (6th edition, 2002) (26) and received trastuzumab as short-term preoperative monotherapy as part of the Unicancer GEP04 RADHER phase II clinical trial (European Clinical Trials Database no., 2007-004098-24). Trastuzumab was administered intravenously following a weekly schedule with a loading dose of $4 \mathrm{mg} / \mathrm{kg}$ at day 1 then $2 \mathrm{mg} / \mathrm{kg} /$ week for 6 weeks. Surgery was performed subsequent to the completion of the sixth cycle. All patients provided written consent, and the study was approved by the Comité de Protection des Personnes Ouest IV (Nantes, France). All patients had baseline 18G-needle biopsies prior to the initiation of the treatment. A second biopsy was performed at surgery. Samples were snap-frozen in liquid nitrogen at $-80^{\circ} \mathrm{C}$ to avoid the degradation of phosphoproteins. Prior to being submitted to total protein extraction, all biopsies were validated by a senior pathologist from the Department of Biopathology of the Institut de Cancérologie de Lorraine (Vandœuvre-lès-Nancy, France) using a hematoxylin-eosin frozen section examination to ensure that the minimal tumor cell content was $>50 \%$.

In total, 19 pairs of baseline/surgery biopsies were eligible for protein extraction and comparative phosphoprotein expression analysis prior to and subsequent to treatment.

Phosphoprotein expression analysis using a multiplex bead immunoassay. The expression levels of p-AKT, phosphorylated-ribosomal protein S6 kinase B1 (p-P70S6K), phosphorylated-mitogen-activated protein kinase kinase 1 (p-MEK1) and phosphorylated-extracellular signal regulated kinase 1/2 (p-ERK1/2) were quantitatively assessed using a multiplex bead immunoassay, as previously described (27). Briefly, proteins were extracted from cell cultures and tumor specimens using cell lysis kit (Bio-Rad Laboratories, Inc., Hercules, CA, USA), according to the manufacturer's instructions. Cell pellets (1x10 ${ }^{6}$ cells), or tumor specimens $(15-20 \mathrm{mg})$ were first disrupted for $15 \mathrm{~min}$ by use a steel-bead tissue lyser (Qiagen $\mathrm{GmbH}$, Hilden, Germany). Disrupted tissues were then exposed for $10 \mathrm{sec}$ to the lysis solution containing phenylmethylsulfonylfluoride antiprotease. Protein extracts were finally stored at $-80^{\circ} \mathrm{C}$ until analyzed.

Proteins extracts were transferred into 96-well plates and diluted with $25 \mu \mathrm{l}$ buffered solution. Multiplexing Bio-Plex phosphoprotein singleplex assay kits (Bio-Rad Laboratories Inc.) for p-AKT (Ser473; catalog no. 171-V1075), p-P70S6K (Thr421/ Ser424; catalog no. 171-V24155), p-MEK1 (Ser217/Ser221; catalog no. 171-V25340) and p-ERK1/2 (Thr202/Tyr204, Thr185/ Tyr187; catalog no. 171-V22238) monoclonal antibodies was performed according to the manufacturer's instructions. First, beads bearing antibodies directed against the phosphoproteins of interest were added to each well and incubated overnight at $37^{\circ} \mathrm{C}$. Biotinylated antibodies and streptavidin phycoerythrin solution were then added. Phosphoprotein expression was then assessed using BioPlex ${ }^{\circledR}$ (Bio-Rad Laboratories, Inc.), in accordance with the manufacturer's instructions. Frozen protein extracts from the MCF-7 cell line exposed to epidermal growth factor (EGF) were used as positive controls. The results were recorded as mean fluorescence intensities, expressed as arbitrary units and considered significant when exceeding a signal:noise ratio of 3. Results were normalized to the data measured in the positive controls.

Statistical analysis. Results were recorded as the mean fluorescence intensity of triplicate experiments (cell lines) or duplicate measurements (patients) and expressed as arbitrary units. Parameters were rescaled by dividing by the mean of the parameter prior to exposure to trastuzumab. Hence, all 
Table I. Expression level of phosphoproteins in HER2-positive SKBR3 and HER2-negative MCF-7 cell lines.

\begin{tabular}{|c|c|c|c|}
\hline \multirow[b]{2}{*}{ Phosphoprotein } & \multicolumn{2}{|c|}{ Expression level } & \multirow[b]{2}{*}{ P-value } \\
\hline & $\begin{array}{l}0 \times 10^{-6} \mathrm{~mol} / 1 \\
\text { trastuzumab }\end{array}$ & $\begin{array}{l}5 \times 10^{-6} \mathrm{~mol} / 1 \\
\text { trastuzumab }\end{array}$ & \\
\hline \multicolumn{4}{|l|}{ MCF-7 } \\
\hline $\mathrm{p}-\mathrm{AKT}$ & $1.00(0.06)$ & $0.95(0.04)$ & 0.36 \\
\hline p-ERK1/2 & $1.00(0.03)$ & $0.96(0.21)$ & 0.78 \\
\hline p-P70S6K & $1.00(0.05)$ & $0.96(0.11)$ & 0.63 \\
\hline p-MEK1 & $1.00(0.13)$ & $0.85(0.17)$ & 0.30 \\
\hline \multicolumn{4}{|l|}{ SKBR-3 } \\
\hline $\mathrm{p}-\mathrm{AKT}$ & $1.00(0.04)$ & $0.32(0.07)$ & $<0.01$ \\
\hline $\mathrm{p}-\mathrm{ERK} 1 / 2$ & $1.00(0.13)$ & $0.68(0.09)$ & 0.02 \\
\hline p-P70S6K & $1.00(0.01)$ & $0.83(0.00)$ & $<0.01$ \\
\hline p-MEK1 & $1.00(0.12)$ & $0.68(0.07)$ & 0.02 \\
\hline
\end{tabular}

Results are expressed as mean (standard deviation). HER2, human epidermal growth factor receptor 2; p-, phosphorylated; AKT, protein kinase B; ERK1/2, extracellular signal regulated kinase 1/2; P70S6K, ribosomal protein S6 kinase B1; MEK, mitogen-activated protein kinase kinase 1.

Table II. Expression level of phosphoproteins in patients prior to and subsequent to treatment with trastuzumab.

Expression level

\begin{tabular}{lccc}
\cline { 2 - 3 } Phosphoprotein & Pre-treatment & Post-treatment & P-value \\
\hline p-AKT & $1.00(1.13)$ & $0.89(1.77)$ & 0.77 \\
p-P70S6K & $1.00(0.24)$ & $0.92(0.15)$ & 0.16 \\
p-MEK1 & $1.00(0.62)$ & $0.98(0.70)$ & 0.92 \\
p-ERK1/2 & $1.00(0.83)$ & $1.00(0.69)$ & 0.99 \\
\hline
\end{tabular}

Results expressed as mean (standard deviation). p-, phosphorylated; AKT, protein kinase B; P70S6K, ribosomal protein S6 kinase B1; MEK, mitogen activated protein kinase kinase 1; ERK1/2, extracellular signal regulated kinase $1 / 2$.

parameters prior to exposure to trastuzumab had a mean that was equal to 1 , and the size of the effect was not affected by this transformation.

Comparisons of the parameters between the two times (baseline and surgery) were made with a paired Student's $t$-test. Statistical analyses were performed using SAS software version 9.2 (SAS Institute Inc., Cary, NC, USA). A P-value of 0.05 was considered to indicate a statistically significant difference.

\section{Results}

In the MCF-7 cell line, the mean expression levels for phosphoproteins following exposure to $5 \times 10^{-6} \mathrm{~mol} / 1$ trastuzumab were 0.95 [standard deviation (SD), 0.04], 0.96 (SD, 0.21), $0.96(\mathrm{SD}, 0.11)$ and $0.85(\mathrm{SD}, 0.17)$ for p-AKT, p-ERK1/2,
A

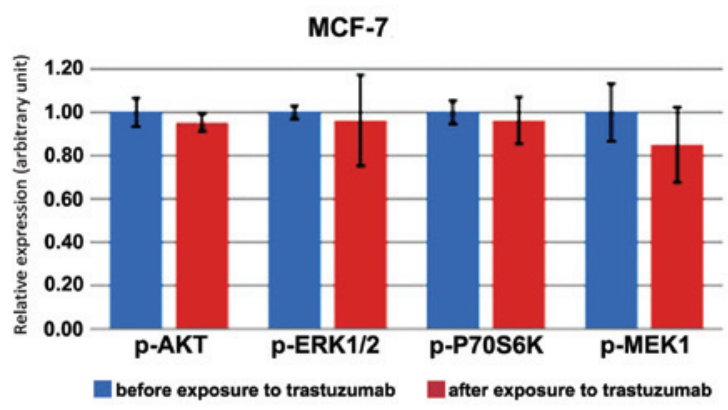

B

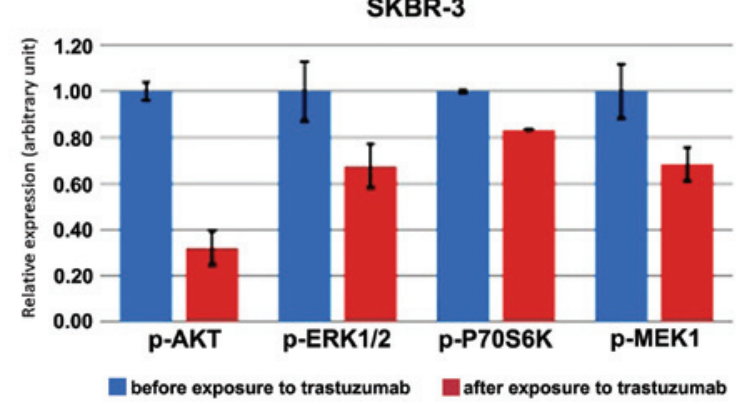

Figure 1. Histograms showing the mean \pm standard deviation (error bars) of phosphoprotein expression. (A) Expression level of phosphoproteins in HER2-negative MCF-7 cell lines prior to exposure to trastuzumab (in blue), and following exposure to trastuzumab (in red). (B) Expression level of phosphoproteins in HER2-positive SKBR3 cell lines prior to exposure to trastuzumab (in blue), and following exposure to trastuzumab (in red). HER2, human epidermal growth factor receptor 2; p-, phosphorylated; AKT, protein kinase B; ERK1/2, extracellular signal regulated kinase 1/2; P70S6K, ribosomal protein S6 kinase B1; MEK, mitogen-activated protein kinase kinase 1.

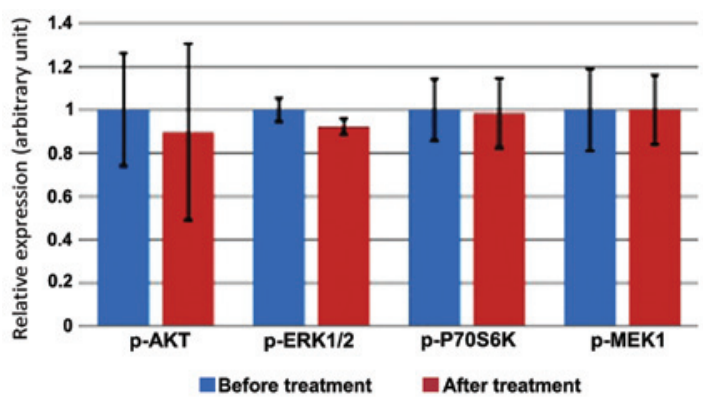

Figure 2. Expression level of phosphoproteins in the clinical specimens of invasive breast cancers, prior to (in blue) and subsequent to (in red) treatment with trastuzumab. Histogram shows the mean \pm standard deviation (error bars) of phosphoprotein expression. p-, phosphorylated; AKT, protein kinase B; ERK1/2, extracellular signal regulated kinase 1/2; P70S6K, ribosomal protein S6 kinase B1; MEK, mitogen-activated protein kinase kinase 1.

P-70S6K and p-MEK1, respectively. No statistically significant difference in the expression level of phosphoproteins prior to and subsequent to exposure to trastuzumab for p-AKT, p-ERK1/2, p-S70S6K and p-MEK1 $(\mathrm{P}=0.36, \mathrm{P}=0.78$, $\mathrm{P}=0.63$ and $\mathrm{P}=0.30$, respectively) was detected in MCF-7 cells (Table I; Fig. 1A).

In the SKBR-3 cell line, the mean expression levels for phosphoproteins following exposure to $5.10^{-6} \mathrm{~mol} / \mathrm{l}$ trastuzumab were 0.32 (SD, 0.07), 0.68 (SD, 0.09), 0.83 (SD, 0.00) and 0.68 (SD, 0.07) for p-AKT, p-ERK1/2, P-70S6K and $\mathrm{p}$-MEK1, respectively. A statistically significant decrease in the expression level of p-AKT $(\mathrm{P}<0.01)$, p-ERK 1/2 $(\mathrm{P}=0.02)$, 
P-70S6K $(\mathrm{P}<0.01)$ and p-MEK1 $(\mathrm{P}=0.02)$ was observed following exposure to trastuzumab in SKBR-3 cells (Table I; Fig. 1B).

In patients, the mean expression levels of phosphoproteins in the biopsies obtained subsequent to treatment were 0.89 (SD, 1.77), 0.92 (SD, 0.15), 0.98 (SD, 0.70) and 1.00 (SD, 0.69) for p-AKT, p-P70S6K, p-MEK1 and p-ERK1/2, respectively. No statistically significant difference in the expression of the p-AKT $(\mathrm{P}=0.77)$, p-P70S6K $(\mathrm{P}=0.16)$, p-MEK1 $(\mathrm{P}=0.92)$ and $\mathrm{p}-\mathrm{ERK} 1 / 2(\mathrm{P}=0.99)$ signaling phosphoproteins was observed subsequent to treatment (Table II; Fig. 2).

\section{Discussion}

Trastuzumab undoubtedly provides a major advancement in the treatment of breast cancer in metastatic or adjuvant settings and alone or in combination with chemotherapy. Trastuzumab, a mAb, dramatically improves the response rate and progression-free and overall survival of patients with metastatic disease (15). However, the exact mode of action of trastuzumab remains partly elucidated, and several studies have suggested that the inhibition of oncogenic signaling may not be the main mode of action $(21,22)$, in particular when trastuzumab is administered alone for chemotherapy-naïve patients $(25,28)$.

In the present study, the expression level of phosphoproteins present in the PI3K/AKT/mTOR and RAS/RAF/MAPK signaling pathways was analyzed prior to and subsequent to exposure to trastuzumab.

The impact of trastuzumab was assessed in cell lines and tumor specimens, which were obtained from patients with HER2-overexpressing early breast cancer that had received trastuzumab as a preoperative monotherapy as part of the RADHER clinical trial.

The present study showed that trastuzumab has a varied impact on the signaling pathways of the MCF-7 and SKBR-3 cell lines. Following exposure to trastuzumab, a statistically significant decrease in the expression levels of p-AKT, p-P70S6K, p-MEK1 and p-ERK1/2 was found in the SKBR-3 cell line, but not in MCF-7 cells. This result was predictable, and may be explained by the fact that SKBR-3 cell line overexpress HER-2, in contrast to the MCF-7 cell line. Notably, no decrease in the expression level of phosphoproteins was observed following treatment with trastuzumab monotherapy in HER2-positive breast cancer patients, which suggests that other mechanisms may interfere with the expression.

Previous studies have demonstrated that trastuzumab has several modes of action, implying that various mechanisms for antitumor activity are present in vitro and in vivo $(18,21,22)$. The modulation of signaling pathways was also shown to be a major mechanism when cell lines were treated with trastuzumab, whereas ADCC was the leading mechanism in small animals treated with the same molecule $(28,29)$. The mechanism of action involved was also shown to vary depending on the neoadjuvant or adjuvant setting, dose and administration schedule and the presence or absence of co-administered drugs $(28,30,31)$. In 2004, Gennari et al showed that trastuzumab employed the preferential immunological mechanism of ADCC when used as a monotherapy and in a preoperative setting (28). Therefore, the classification of the tumor would not be the only parameter to consider when predicting the response to treatment (31).

In the present study, trastuzumab was used in a preoperative setting as a monotherapy; therefore, the lack of downregulation of the PI3K and MAPK pathways could probably be explained by the implementation of a predominant immunological mechanism for a trastuzumab-associated ADCC, which is consistent with previous studies (28). Additional studies are required to elucidate the mechanisms underlying ADCC.

Signaling pathways have been previously shown to be highly interconnected, with multiple points of convergence, cross talk and feedback loops, and these pathways can activate or inhibit each other, depending on the context and on cellular conditions (32-36). A compensatory mechanism, due to the presence of other signaling pathways, could explain the absence of or decrease in the expression of phosphoproteins. However, it should be noted that the present study was performed on a small population, which may have led to a bias in the results. These results should be confirmed in a larger and more representative effective.

Bio-Plex ${ }^{\circledR}$ phosphoprotein arrays do not provide subcellular localization data or take into account the heterogeneity of the tumor tissue (27). Tumor heterogeneity is a major source of variability in results. Tumors can be polyclonal, and will therefore not express homogeneous phosphoproteins. For this reason, it is uncertain whether the samples analyzed were fully representative of the whole tumor, as the stroma is present in various proportions in the tumor tissue. This possibility may explain the difference in the results observed with cell lines that are not affected by the stroma or tumor heterogeneity.

Results can also be affected by parameters such as the pre-analytical phase. The pre-analytical steps for sampling may importantly affect the results, particularly with regards to phosphoproteins (37). Phosphoproteins are very sensitive to temperature and rapidly degrade, which may have an impact on the results. Numerous variables can affect the quality of sampling, particularly intraoperative hypoxia and the cold ischemic time. A previously study on uterus surgical specimens revealed that the time between excision and analysis has an impact on the level of expression of phosphoproteins (38).

The hot and cold ischemia time can affect the quality of samples and induce variation in the expression of phosphoproteins, as phosphoproteins are very sensitive to phosphatase (38). Preventing the dephosphorylation of the marker remains a challenge. In addition, the degradation of phosphoproteins is preceded by an increase of their concentration in the sample. All these parameters are challenging to control. Rapid processing is essential for assessing signaling activity in phosphoprotein studies. In the present study, the processing time has not been traced, which may provide a significant bias in the results. However, the excision and snap-freezing procedure used in the present study ensures little variation in the expression of phosphoproteins, by limiting degradation.

In summary, the degradation of phosphoproteins can vary according to the tissue, tumor type or the phosphoprotein itself (37). Phosphoprotein expression can also be affected by other cellular stressors prior to tissue congelation (10).

Although trastuzumab is widely used for the treatment of HER2-overexpressing breast cancers, the underlying in vivo mechanism of action is not yet fully understood. The present 
study showed that trastuzumab inhibits the MAPK and PI3K pathways for cell lines overexpressing HER2, but not on HER2-overexpressing tumors that have received preoperative treatment as a monotherapy. According to the results, the inhibition of oncogenic signaling pathways may not be the main mode of action employed, when trastuzumab is administered alone in chemotherapy naïve patients. Alternative molecular mechanisms, such as the modulation of immune pathways, should be considered.

\section{References}

1. Hudis CA: Trastuzumab-mechanism of action and use in clinical practice. N Engl J Med 357: 39-51, 2007.

2. Baselga J: Treatment of HER2-overexpressing breast cancer. Ann Oncol 21 (Suppl 7): vii36-vii40, 2010.

3. Engelman JA, Luo J and Cantley LC: The evolution of phosphatidylinositol 3-kinases as regulators of growth and metabolism. Nat Rev Genet 7: 606-619, 2006.

4. Harlé A, Lion M, Lozano N, Husson M, Harter V, Genin P and Merlin JL: Analysis of PIK3CA exon 9 and 20 mutations in breast cancers using PCR-HRM and PCR-ARMS: Correlation with clinicopathological criteria. Oncol Rep 29: 1043-1052, 2013.

5. Mendoza MC, ErEE and Blenis J: The Ras-ERK and PI3K-mTOR pathways: Cross-talk and compensation. Trends Biochem Sci 36: 320-328, 2011.

6. Martelli AM, Tabellini G, Bressanin D, Ognibene A, Goto K, Cocco L and Evangelisti C: The emerging multiple roles of nuclear Akt. Biochim Biophys Acta 1823: 2168-2178, 2012.

7. Park SS and Kim SW: Activated Akt signaling pathway in invasive ductal carcinoma of the breast: Correlation with HER2 overexpression. Oncol Rep 18: 139-143, 2007.

8. Lin HJ, Hsieh FC, Song H and Lin J: Elevated phosphorylation and activation of PDK-1/AKT pathway in human breast cancer. Br J Cancer 93: 1372-1381, 2005.

9. Baker AF, Dragovich T, Ihle NT, Williams R, Fenoglio-Preiser C and Powis G: Stability of phosphoprotein as a biological marker of tumor signaling. Clin Cancer Res 11: 4338-4340, 2005.

10. David KA and Juhl H: Immunohistochemical detection of phosphoproteins and cancer pathways. In: Handbook of Practical Immunohistochemistry. Lin F and Prichard J (eds). Springer, New York, NY, pp85-90, 2015.

11. Mandell JW: Phosphorylation state-specific antibodies: Applications in investigative and diagnostic pathology. Am J Pathol 163: 1687-1698, 2003.

12. Mandell JW: Immunohistochemical assessment of protein phosphorylation state: The dream and the reality. Histochem Cell Biol 130: 465-471, 2008.

13. Menard S, Pupa SM, Campiglio M and Tagliabue E: Biologic and therapeutic role of HER2 in cancer. Oncogene 22: 6570-6578, 2003.

14. Yersal O and Barutca S: Biological subtypes of breast cancer: Prognostic and therapeutic implications. World J Clin Oncol 5: 412-424, 2014

15. Slamon DJ, Leyland-Jones B, Shak S, Fuchs H, Paton V, Bajamonde A, Fleming T, Eiermann W, Wolter J, Pegram M, et al: Use of chemotherapy plus a monoclonal antibody against HER2 for metastatic breast cancer that overexpresses HER2. N Engl J Med 344: 783-792, 2001.

16. Valabrega G, Montemurro F and Aglietta M: Trastuzumab: Mechanism of action, resistance and future perspectives in HER2-overexpressing breast cancer. Ann Oncol 18: 977-984, 2007.

17. Gemmete JJ and Mukherji SK: Trastuzumab (herceptin). AJNR Am J Neuroradiol 32: 1373-1374, 2011.

18. Yakes FM, Chinratanalab W, Ritter CA, King W, Seelig S and Arteaga CL: Herceptin-induced inhibition of phosphatidylinositol-3 kinase and Akt Is required for antibody-mediated effects on p27, cyclin D1, and antitumor action. Cancer Res 62: 4132-4141, 2002.

19. Mohsin SK, Weiss HL, Gutierrez MC, Chamness GC, Schiff R, Digiovanna MP, Wang CX, Hilsenbeck SG, Osborne CK, Allred DC, et al: Neoadjuvant trastuzumab induces apoptosis in primary breast cancers. J Clin Oncol 23: 2460-2468, 2005.
20. Nahta R, Yu D, Hung MC, Hortobagyi GN and Esteva FJ: Mechanisms of disease: Understanding resistance to HER2-targeted therapy in human breast cancer. Nat Clin Pract Oncol 3: 269-280, 2006.

21. Arnould L, Gelly M, Penault-Llorca F, Benoit L, Bonnetain F, Migeon C, Cabaret V, Fermeaux V, Bertheau P, Garnier J, et al: Trastuzumab-based treatment of HER2-positive breast cancer: An antibody-dependent cellular cytotoxicity mechanism? Br J Cancer 94: 259-267, 2006.

22. Clynes RA, Towers TL, Presta LG and Ravetch JV: Inhibitory Fc receptors modulate in vivo cytotoxicity against tumor targets. Nat Med 6: 443-446, 2000.

23. Spector NL and Blackwell KL: Understanding the mechanisms behind trastuzumab therapy for human epidermal growth factor receptor 2-positive breast cancer. J Clin Oncol 27: 5838-5847, 2009.

24. Molina MA, Codony-Servat J, Albanell J, Rojo F, Arribas J and Baselga J: Trastuzumab (herceptin), a humanized anti-Her2 receptor monoclonal antibody, inhibits basal and activated Her2 ectodomain cleavage in breast cancer cells. Cancer Res 61: 4744-4749, 2001

25. Andre F, Dieci MV, Dubsky P, Sotiriou C, Curigliano G, Denkert $\mathrm{C}$ and Loi S: Molecular pathways: Involvement of immune pathways in the therapeutic response and outcome in breast cancer. Clin Cancer Res 19: 28-33, 2013.

26. Sobin LH: TNM, sixth edition: New developments in general concepts and rules. Semin Surg Oncol 21: 19-22, 2003.

27. Chergui F, Chrétien AS, Bouali S, Ramacci C, Rouyer M, Bastogne T, Genin P, Leroux A and Merlin JL: Validation of a phosphoprotein array assay for characterization of human tyrosine kinase receptor downstream signaling in breast cancer. Clin Chem 55: 1327-1336, 2009.

28. GennariR,Menard S, FagnoniF, PonchioL, Scelsi M, Tagliabue E, Castiglioni F, Villani L, Magalotti C, Gibelli N, et al: Pilot study of the mechanism of action of preoperative trastuzumab in patients with primary operable breast tumors overexpressing HER2. Clin Cancer Res 10: 5650-5655, 2004.

29. Therasse P: Measuring the clinical response. What does it mean? Eur J Cancer 38: 1817-1823, 2002.

30. Petricevic B, Laengle J, Singer J, Sachet M, Fazekas J, Steger G, Bartsch R, Jensen-Jarolim E and Bergmann M: Trastuzumab mediates antibody-dependent cell-mediated cytotoxicity and phagocytosis to the same extent in both adjuvant and metastatic HER2/neu breast cancer patients. J Transl Med 11: 307, 2013.

31. Tagliabue E, Campiglio M, Pupa SM, Ménard S and Balsari A: Activity and resistance of trastuzumab according to different clinical settings. Cancer Treat Rev 38: 212-217, 2012.

32. Singh A, Nunes JJ and Ateeq B: Role and therapeutic potential of G-protein coupled receptors in breast cancer progression and metastases. Eur J Pharmacol 763: 178-183, 2015.

33. Hollestelle A, Elstrodt F, Nagel JH, Kallemeijn WW and Schutte M: Phosphatidylinositol-3-OH kinase or RAS pathway mutations in human breast cancer cell lines. Mol Cancer Res 5: 195-201, 2007.

34. Aksamitiene E, Kiyatkin A and Kholodenko BN: Cross-talk between mitogenic Ras/MAPK and survival PI3K/Akt pathways: A fine balance. Biochem Soc Trans 40: 139-146, 2012.

35. Saini KS, Loi S, de Azambuja E, Metzger-Filho O, Saini ML, Ignatiadis M, Dancey JE and Piccart-Gebhart MJ: Targeting the PI3K/AKT/mTOR and Raf/MEK/ERK pathways in the treatment of breast cancer. Cancer Treat Rev 39: 935-946, 2013.

36. Carracedo A and Pandolfi PP: The PTEN-PI3K pathway: Of feedbacks and cross-talks. Oncogene 27: 5527-5541, 2008.

37. Pinhel IF, Macneill FA, Hills MJ, Salter J, Detre S, A'hern R, Nerurkar A, Osin P, Smith IE and Dowsett M: Extreme loss of immunoreactive p-Akt and p-Erk1/2 during routine fixation of primary breast cancer. Breast Cancer Res 12: R76, 2010.

38. Siddiqui S and Rimm DL: Pre-analytic variables and phospho-specific antibodies: The Achilles heel of immunohistochemistry. Breast Cancer Res 12: 113, 2010. 